\title{
Familial Mediterranean fever with a single MEFV mutation: can a deletion resulting in $\alpha$-thalassemia be the cause?
}

Journal of Human Genetics (2011) 56, 169-171; doi:10.1038/jhg.2010.160; published online 23 December 2010

Familial Mediterranean fever (FMF) is an important chronic disease with its clinical course and complications. It affects societies native to the Mediterranean basin, primarily the Turks, Jews, Arabs and Armenians. The MEFV gene (MEFV) on chromosome 16 p13.3 is responsible for this autosomal recessive disease, and the clinical symptoms are expected to be seen in individuals with two mutated alleles. However, there is a symptomatic patient population with only one mutation (25\% of symptomatic cases). This has always been a subject of concern, and there have been studies and reviews undertaken in an effort to explain this situation. ${ }^{1-4}$ General opinion points to the possible presence of a second mutation that has not been revealed with the present methods and remains undefined. There has been no discussion of the possibility that it could be caused by any clinical condition other than the disease itself. However, our recent experiences suggest that concomitant $\alpha$-thalassemia might be one such contributing factor in symptomatic patients who carry only one mutation. In this report, we wish to draw attention to the coexistence of these two diseases that affect the same societies and have overlapping genetic localizations, as a possible alternative explanation for how FMF can occur with a single mutation.

$\alpha$-Thalassemia is an inherited anemia. ${ }^{5}$ It is the commonest monogenic disorder and is frequent in Mediterranean countries. It results from defective synthesis of the $\alpha$ globin chains that constitute adult hemoglobin. Its gene localization is $16 \mathrm{p} 13.3$. This disorder, unlike the other forms of thalassemia, commonly arises from deletions of $\alpha$ globin genes, of which there are four in the normal genome. The clinical manifestations are silent carrier (only one $\alpha$-globin gene is deleted, normal hematological parameters), $\alpha$-thalassemia trait (two genes are missing, microcytic anemia), hemoglobin $\mathrm{H}(\mathrm{HbH})$ disease (three genes are affected, microcytic hypochromic hemolytic anemia) and fatal hydrops fetalis (all four genes are missing).

The index case that launched our interest in the subject was a girl child with microcytic anemia. The combination of reduced red cell indices (Hb $11.7 \mathrm{~g}$ per $100 \mathrm{ml}$, mean corpuscular volume (MCV) $61.7 \mathrm{fl}$, mean corpuscular hemoglobin $(\mathrm{MCH}) 19.2 \mathrm{pg}$ ) with normal body iron status, normal $\mathrm{Hb}$ analysis ( $\mathrm{HbF}$ $0.1 \%$ and $\mathrm{HbA}_{2} 2.2 \%$ ) and demonstrable $\mathrm{HbH}$ inclusion bodies was consistent with $\alpha$-thalassemia trait. Her medical records showed that she had recurrent abdominal pain and was heterozygous for the FMF mutation of E148Q. Investigation of the core family revealed that the patient's mother also had $\alpha$-thalassemia trait, was heterozygous for E148Q and had a history of abdominal pain. In the extended family investigation, the presence of accompanying $\alpha$-thalassemia in every individual with FMF mutation drew particular attention, suggesting that a relation between $\alpha$-thalassemia and FMF might be present (Figure 1). Interestingly, an aunt had very severe FMF clinically, with a single mutation along with $\alpha$-thalassemia compatible with $\mathrm{HbH}$ disease, which might have resulted from deletions affecting both the $\alpha$-globin gene pairs. In the daughter and mother of this aunt, $\alpha$-thalassemia trait was present in addition to homozygote mutation, but not a considerable FMF clinical picture. The family investigation for deletion assessment demonstrated a deletion in the index case that is not one of the common determinants of $\alpha$ thalassemia. A study to define the full extent of the deletion is underway, using the microtiling method. Following this observation, when all the other cases of $\alpha$-thalassemia were queried regarding FMF, it was learned, surprisingly, that almost all reported inflammatory symptoms, and that there was family history of FMF or positive FMF mutation. The presence of clinical symptoms with a single mutation in those who were positive for FMF mutation stood out as a remarkable finding. This coexistence was not thought as a simple coincidence, because similar coexistence was not observed in cases of $\beta$-thalassemia, though it was observed in a family with $\beta$-thalassemia together with $\alpha$-thalassemia. In addition, there was a previous report of the coexistence of another disease (Rubinstein-Taybi syndrome) developed by microdeletion in the same genetic localization in a symptomatic case with a single FMF mutation. $^{6}$

The MEFV gene encodes pyrin, which is an important modulator of innate immunity. When gene mutation occurs, the protein cannot be properly synthesized, inflammation cannot be regulated, and characteristic inflammatory symptoms develop. To date, disease-causing mutations are considered to be point mutations affecting several exons of the $M E F V$. Deletions may also affect the genes and may disrupt the synthesis of their protein products. It is possible that some of the deletions that result in $\alpha$-thalassemia break genetic material from the neighboring $M E F V$, causing loss of function in the gene. Less likely, the deletions may break the mutated region, causing the mutation to disappear, or may break regulatory elements without affecting the $M E F V$ structurally, thereby reducing gene expression. In this case, clinical symptoms can be observed without determining two mutations. In a recent study, the splicing pattern of $M E F V$ in symptomatic heterozygous patients was examined and a qualitative analysis of $M E F V$ transcripts was conducted. Lack of some exons due to deletions was observed. ${ }^{7}$ Furthermore, there is an 


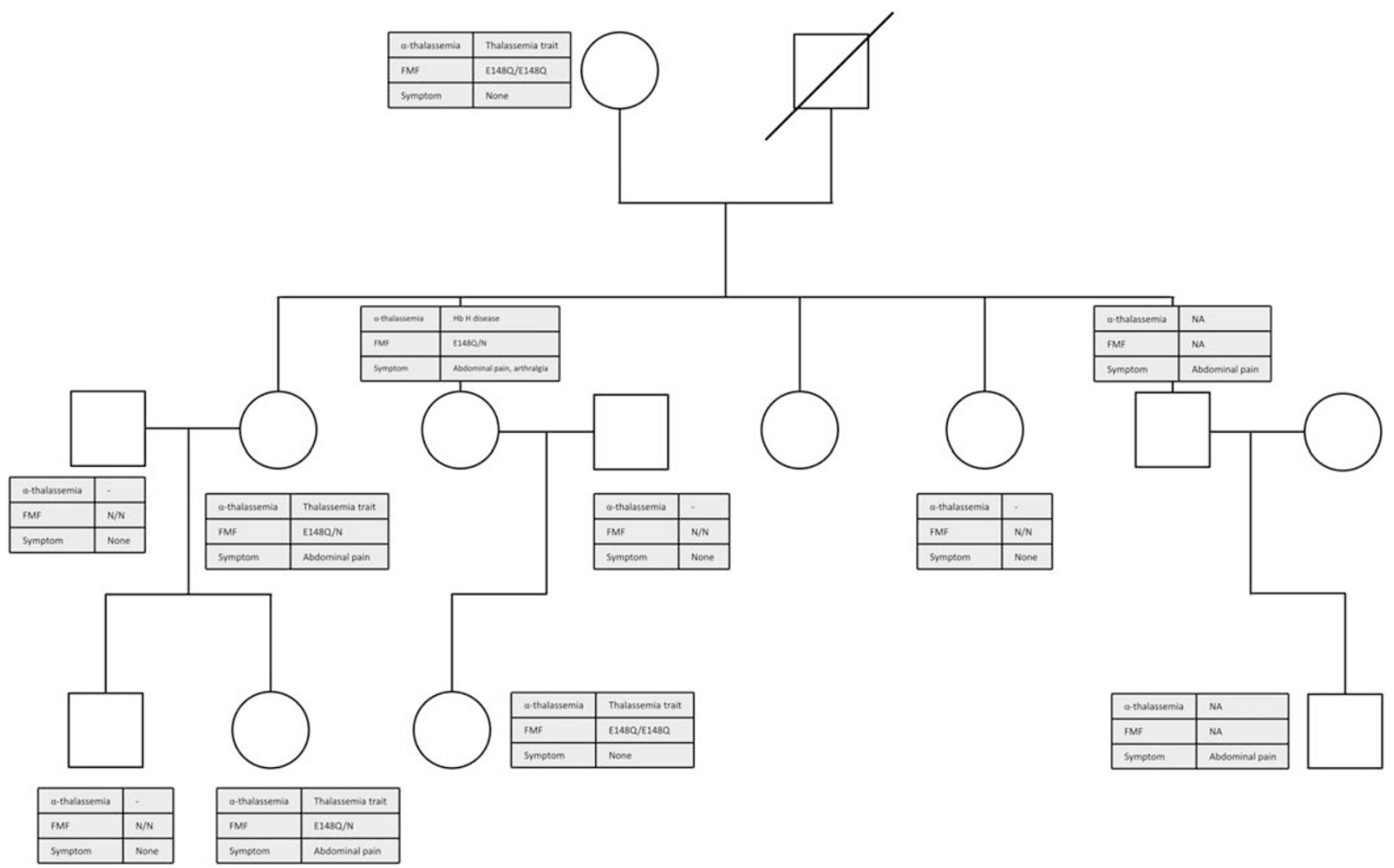

Figure 1 Pedigree from the extended family showing the coexistence of $\alpha$-thalassemia and familial Mediterranean fever. -, studied but not detected; $\mathrm{N}$, negative; NA, not available.

unexplained mild microcytic anemia in FMF cases. One can think of this as anemia of chronic disease (ACD), but cytokine changes that are seen in ACD were not observed in the anemia found in the FMF cases during the diagnosis. ${ }^{8}$ It seems that a deletion hypothesis could explain both symptomatic FMF cases with a single mutation and the microcytic anemia seen in FMF, and, as also previously explained, the genetic defect responsible for the $\alpha$-thalassemia and mental retardation (ATR-16). ${ }^{9}$ The positive results of $M E F V$ transcripts in some controls, regardless of the type of point mutations, also suggest the effect of $\alpha$-thalassemia on MEFV. ${ }^{7}$ The deletion work in our cases will shed light on this matter. Unfortunately, previous studies evaluating FMF disease with a single mutation provided no information regarding even the basic hematological parameters of the patients. However, normal test results do not exclude $\alpha$-thalassemia, as deletion of at least two genes is necessary for hematological symptoms to manifest. It is possible that single-gene deletions that are not detectable by hematological tests or cannot be shown without genetical analysis might have affected the $M E F V$ by a mechanism defined above, or by some other as yet undefined mechanism. As is known, the effect of deletions depends on the critical region they affect rather than their number and size. Briefly, there can be an $\alpha$-globin gene deletion that could affect the functioning of the $M E F V$ even in individuals with normal hematological parameters.

In conclusion, based on our observations and the information available in the literature, we suggest that symptomatic FMF cases with a single mutation should be investigated for $\alpha$-thalassemia. With this brief report, we wish to bring to light the possibility of this association. We believe that simultaneous studies by other interested groups in large patient populations and with advanced genetical laboratory facilities will facilitate clarification of important points such as the following: Which type of $\alpha$-globin gene deletion affects the MEFV? Is the direct or indirect effect of deletions on $M E F V$ different from that of point mutations? For $\alpha$-thalassemia, which has frequent recombination events, can there be intrafamiliar genetic variability? It is our hope that joint efforts will reveal an answer to a question that medical science has for long been endeavoring to resolve.

\section{ACKNOWLEDGEMENTS}

We thank Drs Douglas R Higgs and Chris A Fisher, Weatherall Institute of Molecular Medicine, University of Oxford, for performing the deletion studies.

\section{Deniz Aslan}

Section of Hematology, Department of Pediatrics, Faculty of Medicine, Gazi University, Ankara, Turkey E-mail:drdagutf@ttmail.com and daslan@gazi.edu.tr

1 Moradian, M. M., Sarkisian, T., Ajrapetyan, H. \& Avanesian, N. Genotype-phenotype studies in a large cohort of Armenian patients with familial Mediterranean fever suggest clinical disease with heterozygous MEFV mutations. J. Hum. Genet. 55, 389-393 (2010).

2 Ozen, S. Changing concepts in familial Mediterranean fever: is it possible to have an autosomal-recessive disease with only one mutation? Arthritis Rheum. 60, 1575-1577 (2009). 
3 Booty, M. G., Chae, J. J., Masters, S. L., Remmers, E. F., Barham, B., Le, J. M. et al. Familial Mediterranean fever with a single MEFV mutation: where is the second hit? Arthritis Rheum. 60, 1851-1861 (2009).

4 Marek-Yagel, D., Berkun, Y., Padeh, S., Abu, A., ReznikWolf, H., Livneh, A. et al. Clinical disease among patients heterozygous for familial Mediterranean fever. Arthritis Rheum. 60, 1862-1866 (2009).

5 Higgs, D. R. \& Weatherall, D. J. The alpha thalassaemias. Cell. Mol. Life Sci. 66, 1154-1162 (2009).
6 Kalyoncu, U., Tufan, A., Karadag, O., Kisacik, B., Akdogan, A. \& Calguneri, M. Rubinstein-Taybi syndrome and familial Mediterranean fever in a single patient: two distinct genetic diseases located on chromosome 16p13.3. J. Natl Med. Assoc. 98, 16921693 (2006).

7 Medlej-Hashim, M., Nehme, N., Chouery, E., Jalkh, N. \& Megarbane, A. 1 Novel MEFV transcripts in Familial Mediterranean fever patients and controls. BMC Med. Genet. 11, 87-91 (2010).
8 Celkan, T., Celik, M., Kasapçopur, O., Ozkan, A., Apak, H., Ocak, S. et al. The anemia of familial Mediterranean fever disease. Pediatr. Hematol. Oncol. 22, 657-665 (2005).

9 Wilkie, A. O., Buckle, V. J., Harris, P. C., Lamb, J., Barton, N. J., Reeders, S. T. et al. Clinical features and molecular analysis of the alpha thalassemia/mental retardation syndromes. I. Cases due to deletions involving chromosome band 16p13.3. Am. J. Hum. Genet. 46, 1112-1126 (1990). 